\title{
Edad de inicio en el consumo, motivos y cantidad de alcohol en la determinación de consecuencias en consumidores intensivos universitarios*
}

\author{
Age of Onset, Motives and Amount of Alcohol Consumed in the \\ Determination of Consequences in College Binge Drinkers
}

Recibido: 07 de mayo de 2015| Aceptado: 09 de mayo de 2016

\author{
PATricia Motos Sellés ** \\ María Teresa Cortés TOMÁs *** \\ José Antonio Giménez CostA ***** \\ Universitat de València, España
}

doi: 10.11144/Javeriana.upsy15-2.edcm

Para citar este artículo: Motos Sellés, P., Cortés Tomás, M. A., \& Giménez Costa, J. A. (2016). Edad de inicio en el consumo, motivos y cantidad de alcohol en la determinación de consecuencias en consumidores intensivos universitarios. Universitas Psychologica, 15(2), 243-254. http://dx.doi. org/10.11144/Javeriana.upsy15-2.edcm

* Artículo de investigación científica y tecnológica. Estos resultados forman parte de un proyecto de investigación sobre drogodependencias SPI/3462/2010 financiado por el Ministerio de Sanidad y Política Social de España.

** Correo electrónico: patricia.motos@uv.es

**** Profesora titular de Universidad. Vicedecana de prácticas. Facultad de Psicología. Universitat de València. Correo electrónico: cortesm@uv.es

***** Profesor ayudante doctor. Director de la Unidad de Prevención Comunitaria de Conductas Adictivas. Mancomunidad de la Canal de Navarrés. Correo electrónico: jagimene@uv.es

\section{RES UMEN}

Las implicaciones clínicas y sociales derivadas del consumo intensivo de alcohol (CIA) en universitarios motivan la necesidad de analizar los factores que favorecen su aparición. Este estudio evalúa cómo influye la cantidad de alcohol ingerido, la edad de inicio en el consumo de esta sustancia y los motivos asociados a esta conducta en la presencia de un mayor o menor número de consecuencias psicosociales. 312 estudiantes de primero de la Universidad de Valencia que realizan CIA cumplimentaron el instrumento IECI (Cortés et al., 2012): autoregistro de consumo, motivos asociados a esta ingesta y consecuencias psicosociales derivadas. Se confirman resultados de investigaciones precedentes. Ambos sexos duplican los gramos de alcohol que definen un CIA. Aparecen diferencias en los efectos esperados en función del sexo, así como en la edad de inicio en el consumo y en el número de consecuencias experimentadas. Los análisis de regresión de orden jerárquico muestran la importancia de la edad de inicio y los motivos, por encima de los gramos consumidos, para dar cuenta del deterioro psicosocial generado por la ingesta. Esto apoya la necesidad de atender a la combinación de variables en la explicación y posterior intervención para paliar las consecuencias derivadas del CIA.

Palabras clave

universitarios; edad de inicio; motivos; consecuencias; consumo intensivo de alcohol; diferencias de género

\begin{abstract}
A B S T R A C T
Drinking onset, motives and alcohol use in the prediction of alcohol-related problems in undergraduate binge drinkers. The clinical and social implications derived from binge drinking (BD) in university students cause the need to analyze the factors which contribute to its appearance. This research assesses the influence of the amount of alcohol consumed, drinking onset and the motives associated with this behaviour in the presence of a greater or least number of psychological consequences. 312 first year university students at Valencia University performing BD completed the IECI instrument (Cortés et al., 2012): self report about consumption, associated motives to their consumption and the psychosocial consequences. Results are confirmed by previous research. Both sexes double the grams of alcohol which define a BD. Differences appear in the expected outcome depending on the sex as well as the age at which they drinking onset and the number of experienced consequences. The hierarchical regression analysis shows the importance of the drinking onset and the motives, above the quantity of consumed grams, in order to report the psychosocial
\end{abstract}


impairment generated by the intake. This supports the need to comply with the combination of variables in the explanation and subsequent intervention to alleviate the consequences of the BD.

Keywords

university students; drinking onset; motives; consequences; binge drinking; gender differences

\section{Introducción}

El consumo intensivo de alcohol (CIA) -ingesta de 60 gramos en varones y 40 gramos en mujeres, durante un intervalo de 2-3 horas- (Hingson, Assailly, \& Williams, 2004; MSC, 2008; NIAAA, 2004) es una práctica generalizada entre los jóvenes (Anderson \& Baumberg, 2006) ampliamente representada en población universitaria (Calafat, 2007; March et al., 2010; Parada et al., 2011).

En Europa, dos de cada diez jóvenes (14-24 años) reconocen ingerir alcohol de manera intensiva (European Union, 2010), llegando al 36\% en el caso de los menores de edad españoles (OED, 2013). Además, la prevalencia de consumo intensivo se incrementa con la edad, tal como queda reflejado en el porcentaje de jóvenes que durante el último mes consumieron alcohol -de $57.1 \%$ a los 14 años y a $74.9 \%$ a los 18 años- (OED, 2011). Hay que advertir que a pesar de ser cada vez más homogéneo el número de varones y mujeres que realizan esta ingesta, al evaluar la cantidad de gramos de alcohol consumidos y la frecuencia con la que se hace, en todos los casos los varones muestran mayores índices (Cortés, Espejo, \& Giménez, 2007; Cortés, Giménez, Motos, \& Cadaveira, 2014; O’Malley, \& Johnston, 2002; Wechsler, Dowdall, Davenport, \& Castillo, 1995).

Este patrón de consumo se asocia a múltiples problemas biopsicosociales (Martens et al., 2005; Neighbors, Walker, \& Larimer, 2003; Ray, Turrisi, Abar, \& Peters, 2009), entre los que destacan los síntomas de intoxicación, problemas académicos o profesionales, problemas interpersonales, verse envuelto en conductas sexuales sin protección o no planificadas, conducir bajo los efectos del alcohol, meterse en peleas, sufrir lesiones, tener problemas legales o incluso causar daños a terceros (Cortés, 2010; Hingson, Zha, \& Weitzman, 2009; Kahler, Strong, \& Read, 2005; Mallett et al., 2011; Shield,
Gmel, Patra, \& Rehm, 2012; Wechsler \& Nelson, 2010). Además, se ha demostrado, por un lado, que un aumento en el consumo de alcohol se relaciona también con un mayor número de problemas (Borsari, Neal, Collins, \& Carey, 2001; Cortés et al., 2012) y, por otro lado, que el consumo explica sólo una parte de la varianza de las consecuencias (Borsari et al., 2001; LaBrie \& Pedersen, 2008; Mallett et al., 2011; Neighbors et al., 2003). Por todo ello, la identificación de otros predictores resulta de especial interés.

Neighbors, Lee, Lewis, Fossos, \& Larimer (2007) identificaron los motivos asociados al consumo por parte de los universitarios como uno de los predictores importantes a tener en cuenta. Concretamente, fueron los motivos de afrontamiento ("me ayuda cuando me siento deprimido o nervioso"; "me ayuda a sentirme más seguro de mí mismo", etc.) los que se relacionaron directamente con la aparición de problemas (Conrod, Stewart, Comeau, \& Maclean, 2006; Cooper, 1994; Hauck-Filho, Teixeira, \& Cooper, 2012; Martens, Rocha, Martin, \& Serrao, 2008; McCabe, 2002; Neighbors et al., 2007), mientras que los motivos sociales ("me ayuda a disfrutar de la fiesta"; "me ayuda a relacionarme mejor con los demás", etc.) y de mejora del estado de ánimo ("me produce euforia", "me anima", etc.), que son los predominantes entre consumidores, se relacionaron con la conducta de consumo (Cooper, 1994; Martens et al., 2008; McCabe, 2002).

Además, no puede obviarse que al evaluar los motivos hacia el consumo de alcohol se aprecian, en la mayoría de las ocasiones, diferencias en función del género (Neighbors et al., 2003; Read, Wood, Lejuez, Palfai, \& Slack, 2004; Valdivia \& Stewart, 2005). Los varones recurren al consumo como un mecanismo de afrontamiento, de reconocimiento social y de mejora en las relaciones sexuales (Balodis, Potenza, \& Olmstead, 2009; Harrell \& Karim, 2008; Nolen-Hoeksema, 2004), pero también buscando la embriaguez o los efectos de mejora que asocian al consumo de esta sustancia: euforia, diversión, etc. (Mäkelä \& Mustonen, 2000). Por otro lado, las mujeres buscan mejorar sus relaciones sociales y su capacitación sexual (Ham \& Hope, 2003; Mooney, Fromme, Kivlahan, \& Marlatt, 1987). 
Otro de los factores de riesgo asociado a la aparición de trastornos por consumo de alcohol es la edad en la se inicia la ingesta (Jenkins et al., 2011; Pitkänen, Lyyra, \& Pulkkinen, 2005; Warner, White, \& Johnson, 2007). Al respecto, se encuentran tanto estudios que demuestran esta relación (Bonomo, Bowes, Coffey, Carlin, \& Patton, 2004; Hingson \& Zha, 2009; Livingston, Laslett, \& Dietze, 2008; Pilatti, Caneto, Garimaldi, Del Valle, \& Pautassi, 2013), como otros que la ponen en entredicho, vinculándola con otras variables (Afitska, Plant, Weir, Miller, \& Plant, 2008; Dawson, Goldstein, Chou, Ruan, \& Grant, 2008; Rossow \& Kuntsche, 2013). Esto ha motivado que actualmente se continúe investigando el influjo de la edad de inicio como posible factor que contribuye al incremento en la probabilidad de aparición de consecuencias.

La presente investigación trata de evaluar el peso de la edad de inicio en el consumo de alcohol, los motivos y la cantidad de esta sustancia ingerida en la determinación del número de consecuencias experimentadas por jóvenes universitarios consumidores intensivos. Se considerará en todo momento una perspectiva de género, atendiendo a las diferencias entre varones y mujeres, tanto en la cantidad de consumo como en los motivos asociados al mismo.

\section{Método}

\section{Participantes y procedimiento}

La muestra se ha obtenido a partir de la población de estudiantes de primer curso de cuatro titulaciones de la Universitat de València que aceptaron colaborar en la investigación (Psicología, Logopedia, Trabajo Social y Criminología). En cada una de ellas se seleccionaron dos grupos (uno de mañana y otro de tarde).

La información se recogió mediante un instrumento que los estudiantes autocumplementaron en las aulas, en horario lectivo, estando presente el investigador o algún colaborador entrenado. La participación fue anónima y voluntaria.

Del total de estudiantes encuestados $(n=380)$ se consideran en este estudio sólo los jóvenes entre
17 y 19 años que realizan CIA. La muestra final la componen 312 sujetos ( $66 \%$ mujeres; $34 \%$ varones), con una media de edad de 18.33 años $(D T=0.473)$.

\section{Variables del instrumento}

- Edad de inicio. Los jóvenes indicaron la edad en la que se iniciaron en el consumo de alcohol por decisión propia.

- Patrón de consumo. Se pidió que registraran el número de veces que ingerían alcohol durante los últimos seis meses y en una tabla elaborada ad hoc que anotaran el número de consumiciones ingeridas cada día de una semana de consumo habitual durante esos seis meses, siguiendo un procedimiento similar a Neighbors et al. (2007). También registraban el tipo de alcohol consumido y la hora en la que realizaba cada ingesta. Toda esta información permitió, tomando como referencia las Unidades de Bebida Estándar en España (Rodríguez-Martos, Gual, \& Llopis, 1999), calcular los gramos de alcohol ingeridos en cada consumición. A partir de los mismos se generaron diferentes variables: "suma de gramos diarios", "suma de gramos semanales" y "tipo de consumo" (CIA-NoCIA). Para obtener esta última variable se calcularon los gramos máximos consumidos durante el intervalo de 2 a 3 horas de mayor consumo, etiquetando como consumidor intensivo de alcohol a aquellos chicos que alcanzaban 60 o más gramos de alcohol y a aquellas chicas que alcanzaban $40 \mathrm{o}$ más gramos (MSC, 2008).

- Motivos. Se utilizó el apartado correspondiente a motivos del Instrumento de evaluación de consecuencias derivadas del consumo intensivo de alcohol (IECI) (Cortés et al., 2012), elaborado y validado en población española. Los participantes indicaban en una escala Likert de once puntos si estaban "de acuerdo" (10) o en "desacuerdo" (0) con una serie de dieciséis motivos sobre los posibles efectos buscados al consumir alcohol [Beber ME... (...anima, alegra, produce euforia; ...permite confiar más en mí mismo; ...ayuda a relacionarme con los demás; etc.); Bebo PORQUE... (...es divertido; 
...es habitual hacerlo en ocasiones especiales;... me ayuda a aguantar más tiempo de fiesta; etc.) y Bebo PARA... (...emborracharme; etc.)]. Esta escala presenta buen ajuste en todos los estudios realizados, oscilando el alfa de Cronbach entre 0.900 y 0.913 (Cortés et al., 2012; Motos, 2013).

- Consecuencias. Se evaluaron con el apartado correspondiente del mismo instrumento (IECI) (Cortés et al., 2012). La escala incluye 30 ítems que hacen referencia a síntomas físicos ("he tenido resaca, mareos y vómitos”; etc.); pérdida del control ("he bebido más de lo planeado"; etc.); conductas de riesgo ("he realizado prácticas sexuales de riesgo"; etc.); dependencia física ("necesito mayor cantidad de alcohol que hace unos años"; etc.); autopercepción ("consumir alcohol me hace sentir culpable"; etc.); consecuencias académicas o profesionales ("he descuidado mis responsabilidades a causa de la bebida"; etc.); consecuencias socio-interpersonales ("cuando bebo digo cosas de las que luego me arrepiento"; etc.) y otras consecuencias ("tener problemas económicos a causa de la bebida”; etc.). Todos ellos se responden mediante una escala dicotómica (Sí/No). Tanto para varones como mujeres una puntuación entre 8 y 13 es indicativo de consumo intensivo. Esta escala ha mostrado buen ajuste en estudios previos, alcanzando un alfa de Cronbach superior a 0.807 (Cortés et al., 2012; Motos, 2013).

En el presente estudio se calcula la suma de todas las consecuencias que cada joven señalaba haber presentado en los últimos seis meses, constituyendo la variable "número de consecuencias".

\section{Análisis de datos}

Mediante el paquete estadístico IBM SPSS Statistics 19 se llevaron a cabo análisis descriptivos de las variables de "consumo intensivo", "consumo semanal", motivos hacia al consumo y "número de consecuencias" asociadas al mismo para la muestra general y por sexos. Además, para comprobar la posible existencia de diferencias en función del género se efectuaron comparaciones de medias en estas mismas variables.
A continuación, se examinaron las correlaciones de orden cero entre la suma total de consecuencias, la cantidad de consumo semanal y cada uno de los 16 motivos. Esto permitió confirmar qué elementos estaban más fuertemente asociados con las consecuencias e identificar variables que presentasen relaciones bivariadas imprevistas.

En los análisis posteriores se eliminaron dos de los motivos que no correlacionaron significativamente con el total de consecuencias. A partir de los 14 restantes, se calculó la variable "suma de motivos" para este estudio.

Como paso previo a los análisis de regresión, se aplicó la transformación de Blom (1958) a todas las variables continuas utilizadas en los mismos con el fin de evitar los sesgos en las frecuencias de las medidas y de mantener la coherencia de los datos. Mediante este método los casos son ordenados por rangos, el rango de cada caso se convierte en un percentil y finalmente se normalizan las medidas. El resultado es una puntuación $z$ de rangos, que reduce al mínimo el impacto espurio de los casos extremos.

Se llevaron a cabo tres análisis de regresión de orden jerárquico (uno para el total de la muestra y uno para cada sexo) para detectar las contribuciones únicas de las variables de "gramos semanales" y "suma de motivos" sobre la "suma de consecuencias". Estas dos variables se introdujeron en dos pasos separados (pasos dos y tres). En el primero se introdujo la edad de inicio en el consumo como variable a controlar en la primera etapa, al existir diferencias estadísticamente significativas en función de la misma tanto para el género $[t(305)=$ 2.155, $p=0.032]$, como para el total de "gramos semanales" $[F(8.298)=4.396, p=0.001]$ y la "suma de motivos" $[F(8.298)=2.444, p=0.014]$.

\section{Resultados}

Como recoge la tabla 1 , los varones consumen significativamente más gramos de alcohol a la semana y más gramos de alcohol en una sesión CIA que las mujeres.

Si se atiende a los motivos que presenta este colectivo respecto a su consumo, se aprecia que, en principio, consumen alcohol para mejorar sus 
relaciones sociales (facilita poder hablar con los demás; ayuda a relacionarme con los demás; ayuda a aguantar más tiempo de fiesta) y su estado emocional (anima, alegra, produce euforia; hace sentir bien; es divertido; me gusta la sensación que produce). En segundo lugar destaca el afrontar situaciones estresantes o emociones negativas (hacer o decir cosas que no haría; perder la noción del tiempo).

Únicamente aparecen diferencias significativas por sexos en cuatro de los motivos analizados, dos de ellos con puntuaciones inferiores a 4: "es la manera como se celebran las cosas" ( $p=0.001)$ y "permite arriesgarme más con el sexo" ( $p=0.014)$, ambas con medias superiores en los varones.

También estos últimos manifiestan, significativamente por encima de las mujeres, que consumen alcohol porque lo hacen la mayoría de sus amigos cuando salen de fiesta $(p=0.008)$. En el caso del motivo de mejora de las relaciones sociales, son las mujeres las que reconocen con mayor intensidad que beben alcohol porque les facilita hablar con los demás $(p=0.039)$.

Respecto a las consecuencias asociadas al CIA, la media de problemas experimentados en los últimos seis meses para la muestra total es superior a $9(M=9.86, D T=4.63)$. También en este caso aparecen diferencias significativas entre sexos, siendo los varones los que experimentan mayor número de consecuencias que las mujeres (11.11 frente a 9.19 respectivamente; $t(310)=-3.395, p=0.001$ ).

En la tabla 2 se muestran las correlaciones entre las consecuencias experimentadas, los gramos semanales consumidos y los 16 ítems de la escala de motivos en general y separados por sexos.

La suma del consumo semanal correlaciona positiva y significativamente con el número de consecuencias experimentadas, tanto en el total de la muestra como en el grupo de mujeres.

Del conjunto de motivos, únicamente dos de ellos no correlacionan con el número de con-

TABLA 1.

Motivos hacia el consumo ordenados por su mayor representatividad entre los CIA y diferenciadas por sexos

\begin{tabular}{|c|c|c|c|c|c|c|c|c|}
\hline Ítem & Media & $\mathrm{Sd}$ & Varones & $(\mathrm{M}, \mathrm{sd})$ & Mujeres & $(\mathrm{M}, \mathrm{sd})$ & $\mathrm{t}$ & $\mathrm{p}$ \\
\hline Media de gramos semanales & 164.39 & 113.41 & 228.86 & 141.24 & 131.21 & 77.72 & -7.879 & 0.001 \\
\hline Media de gramos en una sesión CIA & 105.98 & 48.3 & 133.5 & 52.8 & 91.8 & 39.1 & 7.895 & 0.001 \\
\hline Hacer o decir cosas que no haría & 7.07 & 2.622 & 6.72 & 2.858 & 7.26 & 2.479 & 1.653 & 0.100 \\
\hline Anima, alegra, produce euforia & 7.02 & 2.240 & 6.81 & 2.560 & 7.13 & 2.055 & 1.114 & 0.267 \\
\hline Facilita poder hablar con los demás* & 6.53 & 2.807 & 6.08 & 2.851 & 6.77 & 2.761 & 2.072 & 0.039 \\
\hline Es habitual hacerlo en ocasiones especiales & 6.16 & 2.837 & 6.07 & 2.929 & 6.21 & 2.794 & 0.435 & 0.664 \\
\hline Ayuda a relacionarme con los demás & 5.95 & 2.987 & 5.72 & 3.039 & 6.06 & 2.960 & 0.969 & 0.333 \\
\hline ción que prod & 5.89 & 2.639 & & 2.673 & 5.77 & 2.620 & -1.158 & 0.248 \\
\hline Me ay & 5.65 & 3.128 & 5.8 & 3.128 & 5.5 & 3.130 & -0.790 & 0.430 \\
\hline Hace & 5.50 & 2.428 & 5.30 & 2.652 & 5.60 & 2.305 & 0.989 & 0.324 \\
\hline Es di & 5.48 & 2.797 & 5.33 & 2.963 & 5.5 & 2.724 & 0.681 & 0.496 \\
\hline Perder no & 5.37 & 3.037 & 5.07 & 3.276 & 5.52 & 2.903 & 1.263 & 0.207 \\
\hline * la mayoría de mis amigos & 4.84 & 3.333 & 5.54 & 3.178 & 4.48 & 3.361 & -2.680 & 0.008 \\
\hline Para emborracharme & 4.57 & 3.200 & 4.86 & 3.331 & 4.43 & 3.128 & -1.128 & 0.266 \\
\hline Permite c & $4.4^{\prime}$ & 3.012 & & 3.084 & & 2.981 & 0.420 & 0.675 \\
\hline Es la manera como se celebran las cosas** & 3.94 & 3.085 & 4.94 & 3.358 & 3.42 & 2.806 & -4.013 & 0.001 \\
\hline Permite arriesgarme más con el sexo* & 3.82 & 3.377 & 4.47 & 3.508 & 3.49 & 3.265 & -2.463 & 0.014 \\
\hline $\begin{array}{l}\text { Ayuda cuando me siento deprimido o } \\
\text { nervioso }\end{array}$ & 2.95 & 2.842 & & 3.323 & 2.76 & 2.547 & -1.555 & 0.122 \\
\hline Suma de los 16 motivos & 85.22 & 27.15 & 86.58 & 29.29 & 84.52 & 26.03 & -0.631 & 0.528 \\
\hline
\end{tabular}

$* 0.01<\mathrm{p}<0.05 * 0.001<\mathrm{p}<0.01$

Fuente: elaboración propia. 
secuencias experimentadas en la muestra total ("hace sentir bien" y "facilita poder hablar con los demás”), los cuales se eliminaron en los análisis posteriores. Asimismo, entre las mujeres, dos de los motivos no correlacionan con el número de consecuencias. En este caso coincide con la muestra total en el ítem "hace sentir bien", aunque no resulta significativo el hecho de que el alcohol "ayuda cuando se sienten deprimidas o nerviosas".

Para los varones, únicamente seis de los 16 motivos muestran correlaciones significativas: "permite confiar más en mí mismo", "permite arriesgarme más con el sexo", "es divertido", "me ayuda a aguantar más tiempo de fiesta", "me gusta la sensación que produce y para emborracharme".

Las tablas 3, 4 y 5 presentan los resultados de los análisis de regresión de orden jerárquico para predecir las consecuencias derivadas del consumo de alcohol, tanto para la muestra global como para cada uno de los sexos.

La variable edad de inicio incluida en el primer paso de los tres análisis resulta significativa en todos los casos, explicando un $8 \%$ de la varianza para el total de la muestra, un 9.6\% para los varones y un $5.7 \%$ para las mujeres.

La cantidad de alcohol consumida a la semana, introducida en el segundo paso, también resulta significativa para la muestra total y para las mujeres, aunque no para los varones. Para el total, la cantidad de varianza aportada a la predicción de las consecuencias es de $6.1 \%$, y para las mujeres de $5.1 \%$, disminuyendo hasta $2.9 \%$ en el caso de los chicos.

En el último paso se incluye la suma de los 14 motivos que han resultado significativos en la correlación con las consecuencias. En este caso, los porcentajes de varianza en los tres análisis efectuados también resultan significativos. Para la muestra global aporta un $6.3 \%$ de la explicación de las consecuencias. Por sexos la contribución es superior en las chicas (8.3\%) que en los chicos (4.9\%).

Como resumen general de los tres análisis ejecutados, el modelo total aporta a la predicción de la varianza un $20.4 \%$ para la muestra general. Este porcentaje es un punto superior al del subgrupo de las mujeres (19\%) y tres puntos cuando se compara con el de los varones (17.4\%).

TABLA 2.

Correlaciones de los gramos semanales consumidos y los motivos con el número de consecuencias

\begin{tabular}{|llll|}
\hline Medida & Todos & Hombres & Mujeres \\
Gr consumo semanal & $0.245^{* *}$ & 0.165 & $0.209^{* *}$ \\
Perder la noción del tiempo & $0.172^{* *}$ & 0.116 & $0.244^{* *}$ \\
Anima, alegra, produce euforia & $0.161^{* *}$ & 0.112 & $0.231^{* *}$ \\
Hace sentir bien & 0.083 & 0.098 & 0.096 \\
Hacer o decir cosas que no haría & $0.217^{* *}$ & 0.153 & $0.309^{* *}$ \\
Facilita poder hablar con los demás & 0.102 & 0.084 & $0.159^{*}$ \\
Permite confiar más en mi mismo & $0.187^{* *}$ & $0.220^{*}$ & $0.182^{* *}$ \\
Ayuda cuando me siento deprimido o nervioso & $0.166^{* *}$ & 0.174 & 0.131 \\
Permite arriesgarme más con el sexo & $0.352^{* *}$ & $0.369^{* *}$ & $0.312^{* *}$ \\
Ayuda a relacionarme con los demás & $0.173^{* *}$ & 0.118 & $0.236^{* *}$ \\
Es habitual hacerlo en ocasiones especiales & $0.120^{*}$ & 0.089 & $0.154^{*}$ \\
Es divertido & $0.229^{* *}$ & $0.255^{* *}$ & $0.234^{* *}$ \\
Me ayuda a aguantar más tiempo de fiesta & $0.196^{* *}$ & $0.200^{*}$ & $0.188^{* *}$ \\
Es la manera como se celebran las cosas & $0.197^{* *}$ & 0.175 & $0.144^{*}$ \\
Me gusta la sensación que produce & $0.188^{* *}$ & $0.211^{*}$ & $0.159^{*}$ \\
Es lo que hacen la mayoría de mis amigos cuando salimos & $0.185^{* *}$ & 0.127 & $0.181^{* *}$ \\
Para emborracharme & $0.315^{* *}$ & $0.484^{* *}$ & $0.193^{* *}$ \\
\hline
\end{tabular}

$* 0.01<\mathrm{p}<0.050 .01<\mathrm{p}<0.05 * 0.001<\mathrm{p}<0.010 .001<\mathrm{p}<0.01$

Fuente: elaboración propia. 
EDAD DE INICIO EN EL CONSUMO, MOTIVOS Y CANTIDAD DE ALCOHOL EN LA DETERMINACIÓN DE CONSECUENCIAS EN CONSUMIDORES INTENSIVOS UNIVERSITARIOS

TABLA 3.

Análisis de regresión de orden jerárquico para predecir el número de consecuencias derivadas del CIA

\begin{tabular}{|c|c|c|c|c|c|c|c|c|c|c|c|c|}
\hline & & & & & & & \multicolumn{2}{|c|}{ Paso 1} & \multicolumn{2}{|c|}{ Paso 2} & \multicolumn{2}{|c|}{ Paso 3} \\
\hline & $\begin{array}{l}\text { Paso en el modelo de } \\
\text { regresión }\end{array}$ & $\mathrm{R}$ & $\mathrm{R} 2$ & $\begin{array}{l}\text { Error típ. de } \\
\text { estimación }\end{array}$ & $\Delta \mathrm{R}^{2}$ & $\mathrm{~F} \Delta \mathrm{R}^{2}$ & $\mathrm{~b}$ & $\begin{array}{l}\text { Valor de } \\
\text { p parab }\end{array}$ & $\mathrm{b}$ & $\begin{array}{l}\text { Valor de } \\
\text { p para b }\end{array}$ & b & $\begin{array}{l}\text { Valor de } \\
\text { p para b }\end{array}$ \\
\hline ن & Paso 1: edad inicio & 0.283 & 0.080 & 0.952 & 0.080 & $26.466 * *$ & -0.283 & 0.001 & -0.200 & 0.001 & -0.167 & 0.002 \\
\hline 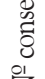 & $\begin{array}{l}\text { Paso 2: Gr consumo } \\
\text { semanal }\end{array}$ & 0.376 & 0.141 & 0.921 & 0.061 & $21.704 * *$ & - & - & 0.261 & 0.001 & 0.195 & 0.001 \\
\hline 乙 & Paso 3: Suma motivos & 0.452 & 0.204 & 0.888 & 0.063 & $23.968 * *$ & - & - & & & 0.264 & 0.001 \\
\hline
\end{tabular}

$* 0.01<\mathrm{p}<0.5 * * 0.001<\mathrm{p}<0.01 * * * \mathrm{p}<0.01 \mathrm{p}<0.001$

TABLA 4.

Análisis de regresión de orden jerárquico en varones para predecir el número de consecuencias derivadas del CIA

\begin{tabular}{|c|c|c|c|c|c|c|c|c|c|c|c|c|}
\hline & & & & & & & \multicolumn{2}{|c|}{ Paso 1} & \multicolumn{2}{|c|}{ Paso 2} & \multicolumn{2}{|c|}{ Paso 3} \\
\hline & $\begin{array}{l}\text { Paso en el modelo de } \\
\text { regresión }\end{array}$ & $\mathrm{R}$ & $R^{2}$ & $\begin{array}{l}\text { Error tip. } \\
\text { de estim. }\end{array}$ & $\Delta R^{2}$ & $F \Delta R^{2}$ & $\beta$ & $\begin{array}{l}\text { Valor de } \\
p \text { para } \beta\end{array}$ & $\beta$ & $\begin{array}{l}\text { Valor de } \\
p \text { para } \beta\end{array}$ & $\beta$ & $\begin{array}{l}\text { Valor de } \\
p \text { para } \beta\end{array}$ \\
\hline$\dot{\bigotimes}$ & Paso 1: edad inicio & 0.310 & 0.096 & 0.990 & 0.096 & $19.770 * *$ & -0.310 & 0.001 & -0.247 & 0.015 & -0.216 & 0.030 \\
\hline $\begin{array}{l}0 \\
0 \\
0\end{array}$ & $\begin{array}{l}\text { Paso 2: Gr consumo } \\
\text { semanal }\end{array}$ & 0.354 & 0.126 & 0.978 & 0.029 & 3.345 & - & - & 0.182 & 0.070 & 0.103 & 0.318 \\
\hline 之 & Paso 3: Suma motivos & 0.417 & 0.174 & 0.956 & 0.049 & $5.819 *$ & - & - & & & 0.240 & 0.018 \\
\hline
\end{tabular}

$* 0.01<\mathrm{p}<0.05 \quad 0.01<\mathrm{p}<0.05 * * 0.001<\mathrm{p}<0.01 \quad 0.001<\mathrm{p}<0.01 * * * \mathrm{p}<0.001 \mathrm{p}<0.001$

TABLA 5.

Análisis de regresión de orden jerárquico en mujeres para predecir el número de consecuencias derivadas del CIA

\begin{tabular}{|c|c|c|c|c|c|c|c|c|c|c|c|c|}
\hline & & & & & & & \multicolumn{2}{|c|}{ Paso 1} & \multicolumn{2}{|c|}{ Paso 2} & \multicolumn{2}{|c|}{ Paso 3} \\
\hline & $\begin{array}{l}\text { Paso en el modelo de } \\
\text { regresión }\end{array}$ & $R$ & $R^{2}$ & $\begin{array}{l}\text { Error tip. } \\
\text { de estim. }\end{array}$ & $\Delta R^{2}$ & $F \Delta R^{2}$ & $\beta$ & $\begin{array}{l}\text { Valor de } \\
\text { p para } \beta\end{array}$ & $\beta$ & $\begin{array}{l}\text { Valor de } \\
p \text { para } \beta\end{array}$ & $\beta$ & $\begin{array}{l}\text { Valor de } \\
p \text { para } \beta\end{array}$ \\
\hline & Paso 1: edad inicio & 0.238 & 0.057 & 0.916 & 0.057 & $12.179^{* * *}$ & -0.238 & 0.001 & -0.177 & 0.011 & -0.145 & 0.030 \\
\hline 造 & $\begin{array}{l}\text { Paso 2: Gr consumo } \\
\text { semanal }\end{array}$ & 0.328 & 0.108 & 0.894 & 0.051 & $11.408 * *$ & - & - & 0.233 & 0.001 & 0.172 & 0.011 \\
\hline 樂 & Paso 3: Suma motivos & 0.436 & 0.190 & 0.853 & 0.083 & $20.372 * * *$ & - & - & & & 0.297 & 0.001 \\
\hline
\end{tabular}

$* 0.01<\mathrm{p}<0.050 .01<\mathrm{p}<0.05 * * * 0.001<\mathrm{p}<0.010 .001<\mathrm{p}<0.01 * * * \mathrm{p}<0.001 \mathrm{p}<0.001$

\section{Discusión y conclusiones}

La Comisión Clínica del Plan Nacional sobre Drogas (2007) señaló como bebedor de riesgo la persona que consume $28 \mathrm{UBE} / \mathrm{semana}$ en el caso de los varones y $17 \mathrm{UBE} /$ semana en las mujeres. En la muestra de jóvenes analizada no se llega a alcanzar estos niveles de ingesta, pero no hay que olvidar que entre los menores de edad cualquier consumo se considera de riesgo (Anderson \& Baumberg, 2006; MSC, 2008), lo que incluye a una parte de la muestra evaluada en este trabajo.
Si analizamos el consumo intensivo, la cantidad de alcohol que ingiere la muestra estudiada dobla el límite establecido por el Ministerio de Sanidad y Consumo (2008) -60g/40g-, coincidiendo con los resultados obtenidos en investigaciones anteriores (Cadaveira, 2010; Cortés, Espejo, \& Giménez, 2008; Cortés, 2012; Cortés et al., 2014; Giménez, 2011; Motos, 2013; White, Kraus, \& Swartzwelder, 2006).

Cuando se atiende a los motivos, en general prima la búsqueda de los efectos de mejora del estado de ánimo y de las relaciones sociales, confirmando nuevamente resultados previos (Hauck-Filho et 
al., 2012; Lyvers, Hasking, Hani, Rhodes, \& Trew, 2009; Martens et al., 2008; Neighbors et al., 2007). Esta realidad señala la necesidad de proponer actividades, independientemente del sexo, que les ayuden a tomar conciencia de la subjetividad con la que evalúan los efectos derivados del consumo de alcohol. Los jóvenes obvian que se trata de una sustancia depresora, con la que difícilmente se podrán obtener estos efectos realizando ingestas tan elevadas.

Aunque se aprecia una homogeneización en la mayoría de los motivos evaluados, destacan todavía los varones en algunos como arriesgarse con el sexo (Balodis et al., 2009; Harrell \& Karim, 2008; Nolen-Hoeksema, 2004) y sucumbir a la presión social (Cortés, 2010). En el caso de las chicas predomina la mejora de las relaciones sociales (Ham \& Hope, 2003; Mooney et al., 1987). Estos resultados apuntan la necesidad de trabajar grosso modo el mismo tipo de motivos en ambos sexos, ya que las diferencias aparecen en variables con menor peso.

Por otra parte, en la muestra general, y en el caso de las mujeres en particular, se confirma una relación positiva y significativa entre la cantidad de alcohol ingerida y el número de consecuencias experimentadas (Martens et al., 2005; Neighbors et al., 2003; Ray et al., 2009). Llama la atención que precisamente entre los varones no se confirme esta relación cuando son los que más consumen y los que mayor número de consecuencias manifiestan. Esto puede ser debido principalmente a dos aspectos: por un lado, a un problema de tamaño muestral, lo que requeriría una mejora en futuras investigaciones, y, por otro lado, a la evidencia de que son un conjunto de variables, no sólo la cantidad de consumo, las que permiten explicar el deterioro psicosocial (Borsari et al., 2001; LaBrie \& Pedersen, 2008; Mallett et al., 2011; Neighbors et al., 2003). Entre ellas, la edad de inicio adquiere importancia para todos los grupos como factor de riesgo, lo que refuerza la investigación precedente que muestra cómo un inicio precoz se vincula con un mayor número de síntomas propios del consumo (Hingson, Zha, \& Wheitzman, 2009; Jenkins et al., 2011; Livingston et al., 2008; Pilatti et al., 2013; Warner et al., 2007). En el caso de los varones, esta variable alcanza más peso que la propia cantidad de alcohol consumida, mientras que en las mujeres el porcentaje explicado es similar en ambas variables.

De ahí la necesidad de potenciar intervenciones en prevención universal que refuercen un retraso en la edad de inicio del consumo de alcohol, haciendo hincapié en aquellas que han mostrado su eficacia, como los controles legales (EMCDDA, 2009). Este tipo de medidas serían recomendables para mujeres y de manera mucho más acentuada para los varones.

Junto con la edad de inicio, los resultados confirman la importancia de los motivos por los que se consume a la hora de explicar las consecuencias de dicha ingesta para ambos sexos, aunque es entre las mujeres donde adquieren mayor peso explicativo, especialmente los motivos de afrontamiento ("hacer o decir cosas que no harían estando sobrias" y "perder la noción del tiempo"). En el caso de los varones también predominan la búsqueda de la embriaguez y la sensación que produce el consumo de esta sustancia.

Estos resultados confirman, en el caso de las mujeres, la investigación precedente (Conrod et al., 2006; Cooper, 1994; Hauck-Filho et al., 2012; Martens et al., 2008; McCabe, 2002; Neighbors, et al., 2007), pero en varones abren una nueva vía de exploración al relacionarse los motivos de mejora con problemas derivados del consumo.

En este sentido, trabajar los motivos es un aspecto a considerar en las actuaciones que buscan paliar las consecuencias del consumo intensivo. Además, es importante apuntar que teóricamente los motivos pueden ser más fácilmente maleables en respuesta a las intervenciones (Martin, 2011). Un reciente meta-análisis que incluye 62 estudios sobre intervenciones realizadas con universitarios consumidores (Carey, Scott, Carey, \& DeMartini, 2007) sugiere que los tratamientos que obvian el cambio de motivos son menos eficaces que los que no lo hacen.

En futuras investigaciones sería importante tratar de incrementar la varianza explicada de la gravedad del consumo (en nuestro caso medido por el número de consecuencias) incluyendo la evaluación de otras variables explicativas, como las nor- 
mas percibidas sobre la ingesta de alcohol por parte del entorno (Neighbors et al., 2007) o variables de personalidad (Carlson \& Johnson, 2012; Cortés et al., 2014), las cuales han mostrado su eficacia de manera independiente.

Asimismo, sería necesario ampliar la muestra no sólo en tamaño y mejor equilibrio entre sexos, sino incluyendo grupos poblacionales diferentes a los universitarios, lo que facilitaría la generalización posterior de resultados.

Este trabajo apoya la investigación que señala la necesidad de tener en cuenta no sólo la cantidad de alcohol ingerida como determinante en la aparición de consecuencias, sino otros factores como los motivos y la edad de inicio en el consumo. Aunque también remarca la necesidad de considerar un mayor número de variables para incrementar el porcentaje de varianza explicada, facilitando, de este modo, un mejor ajuste de la intervención psicosocial a realizar en estos colectivos.

\section{Referencias}

Afitska, N., Plant, M.A., Weir, I., Miller, P., \& Plant, M.L. (2008). The relationship between teenage 'binge' drinking, age of first alcohol consumption and intoxication. J Subst Use, 13, 205-218. http:// dx.doi.org/10.1080/14659890701740663

Anderson, P., \& Baumberg, B. (2006). Alcohol in Europe. London: Institute of Alcohol Studies.

Balodis, I. M., Potenza, M. N., \& Olmstead, M. C. (2009). Binge drinking in undergraduates: relationships with sex, drinking behaviours, impulsivity, and the perceived effects of alcohol. Behavioral Pharmacology, 20, 518-526. http://dx.doi. org/10.1097/FBP.0b013e328330c779

Blom, G. (1958). Statistical estimates and transformed beta-variables. New York: John Wiley and Sons.

Bonomo, Y. A., Bowes, G., Coffey, C., Carlin, J. B., $\&$ Patton, G. C. (2004). Teenage drinking and the onset of alcohol dependence: a cohort study over seven years. Addiction, 99, 1520-1528. http:// dx.doi.org/10.1111/j.1360-0443.2004.00846.x

Borsari, B., Neal, D. J., Collins, S. E., \& Carey, K. B. (2001). Differential Utility of Three Indexes of Risky Drinking for Predicting Alcohol Problems in
College Students. Psychology of Addictive Behaviors, 15(4), 321-324. http://dx.doi.org/10.1037/0893164X.15.4.321

Cadaveira, F. (2010). Consecuencias neurocognitivas: estudio de seguimiento en jóvenes españoles que realizan binge drinking [Neurocognitive Consequences: followup study in Spanish youth binge drinkers]. Trabajo presentado en el Seminario Consumo Intensivo de Alcohol en Jóvenes: conocimiento, alternativas y viabilidad. Mayo, Valencia.

Calafat, A. (2007). El abuso de alcohol de los jóvenes en España [Alcohol abuse by young people in Spain]. Adicciones, 19, 217-224.

Carey, K. B., Scott, L., Carey, M. P., \& DeMartini, K. S. (2007). Individual-level interventions to reduce college student drinking: a meta-analytic review. Addictive Behaviors, 32, 2469-2494. http://dx.doi. org/10.1016/j.addbeh.2007.05.004

Carlson, S. R., \& Johnson, S. C. (2012). Impulsivity is not always associated with student drinking: A moderation study of impulsivity and drinking by positive alcohol expectancies. Addictive Behaviors, 37, 556-560. http://dx.doi.org/10.1016/j. addbeh.2011.12.007

Comisión Clínica de la Delegación del Gobierno para el Plan Nacional Sobre Drogas (2007). Informe sobre alcohol [Report on Alcohol]. Madrid: Ministerio de Sanidad y Consumo.

Conrod, P. J., Stewart, S. H., Comeau, N., \& Maclean, A. M. (2006). Efficacy of Cognitive-Behavioral Interventions Targeting Personality Risk Factors for Youth Alcohol Misuse. Journal of Clinical Child and Adolescent Psychology, 35(4), 550-563. http:// dx.doi.org/10.1207/s15374424jccp3504_6

Cooper, M. L. (1994). Motivations for alcohol use among adolescents: Development and validation of a fourfactor model. Psychological Assessment, 6, 117-128.

Cortés, M. T. (2010). Debates en drogodependencias: Aspectos sociales y de salud en el botellón: obligados a entenderse [Discussions in drug addiction: social aspects and health in the "botellon": required to understand]. Valencia: Plan Municipal de Drogodependencias. Ayuntamiento de Valencia.

Cortés, M. T. (2012). Determinantes psico-sociales relacionados con el consumo intensivo de alcohol de mujeres jóvenes [Psychosocial determinants related 
to intensive alcohol consumption of young women]. Trabajo presentado en las XIV Jornadas asociación Proyecto hombre. Jóvenes y alcohol: enfocando una realidad, Madrid.

Cortés, M. T., Espejo, B., \& Giménez, J. A. (2007). Características que definen el fenómeno del botellón en universitarios y adolescentes [Characteristics that define the phenomenon of the "botellón" in college and adolescents]. Adicciones, 19(4), 357-372.

Cortés, M. T., Espejo, B., \& Giménez, J. A. (2008). Aspectos cognitivos relacionados con la práctica del botellón [Cognitive aspects related to the practice of "botellón"]. Psicothema, 20, 396-402.

Cortés, M. T., Giménez, J. A., Motos, P., \& Cadaveira, F. (2014). The importance of expectations in the relationship between impulsivity and binge drinking among university students. Adicciones, 26(2), 134-145.

Cortés, M. T., Giménez, J. A., Tomás, I., Espejo, B., Pascual, F., Pedrero, E., \& Guardia, J. (2012). Instrumento de Evaluación del Consumo Intensivo de Alcohol [Intensive Alcohol Consumption Assessment Instrument]. Informe final proyecto de investigación presentado al Plan Nacional sobre Drogas.

Dawson, D. A., Goldstein, R. B., Chou, S. P., Ruan, W. J., \& Grant, B. F. (2008). Age at first drink and the first incidence of adult-onset DSM-IV alcohol use disorders. Alcohol Clin Exp Res, 32, 2149-2160. doi: http://dx.doi.org/10.1111/j.15300277.2008.00806.x

European Monitoring Centre for Drugs and Drug Addiction (EMCDDA) (2009). Annual Report 2009.

Recuperado de http://www.emcdda.europa.eu/ attachements.cfm/att_93236_EN_EMCDDA_ AR2009_EN.pdf

European Union (2010). Special Eurobarometer 331: EU citizens' attitudes towards alcohol. Brussels: European Union.

Giménez, J. A. (2011). Análisis de los determinantes cognitivos que subyacen a la conducta de consumo intensivo de alcohol en jóvenes utilizando como marco de referencia la Teoría de la Conducta Planificada [Analysis of cognitive determinants underlying the behavior of heavy alcohol consumption in young people using as a framework the Theory of Planned Behavior] (Tesis doctoral). Valencia: Universitat de Valéncia.
Ham, L. S., \& Hope, D. A. (2003). College students and problematic drinking: A review of the literature. Clinical Psychology Review, 23, 719-759. http:// dx.doi.org/10.1016/S0272-7358(03)00071-0

Harrell, Z. A., \& Karim, N. M. (2008). Is gender relevant only for problem alcohol behaviors? An examination of correlates of alcohol use among college students. Addictive Behavior, 33, 359-365. http:/ dx.doi.org/10.1016/j.addbeh.2007.09.014

Hauck-Filho, N., Teixeira, M. A., \& Cooper M. L. (2012). Confirmatory factor analysis of the Brazilian version of the Drinking Motives Questionnaire-Revised (DMQ-R). Addictive Behavior 37, 524-7. http://dx.doi.org/10.1016/j.addbeh.2011.11.023. Epub 2011 Nov 27.

Hingson, R. W., Assailly, J. P., \& Williams, A. F. (2004). Underage drinking: Frequency, consequences, and interventions. Traffic Injury Prevention, 5, 228-236. http://dx.doi.org/10.1080/15389580490465256

Hingson, R. W., \& Zha, W. (2009). Age of drinking onset, alcohol use disorders, frequent heavy drinking, and unintentionally injuring oneself and others after drinking. Pediatrics, 123, 1477-1484. http:// dx.doi.org/10.1542/peds.2008-2176

Hingson, R., Zha, W., \& Weitzman, E. R. (2009). Magnitude of and trends in alcohol-related mortality and morbidity among US college students ages 18-24. Journal of Study on Alcohol and Drugs, suppl. 16, $12-20$.

Jenkins, M. B., Agrawal, A., Lynskey, M. T., Nelson, E. C., Madden, P. A., Bucholz, K. K., \& Heath, A. C. (2011). Correlates of alcohol abuse/dependence in early-onset alcohol-using women. Am J Addict 20, 429-34. http://dx.doi.org/10.1111/j.15210391.2011.00151.x.

Kahler, C. W., Strong, D. R., \& Read, J. P. (2005). Toward efficient and comprehensive measurement of the alcohol problems continuum in college students: The Brief Young Adult Alcohol Consequences Questionnaire. Alcoholism: Clinical and Experimental Research, 29, 1180-1189. http://dx.doi. org/10.1097/01.ALC.0000171940.95813.A5

LaBrie, J., \& Pedersen, E. (2008). Prepartying promotes heightened risk in the college environment: An event-level report. Addictive Behaviors, 33, 955-959. http://dx.doi.org/10.1016/j.addbeh.2008.02.011 
Livingston, M., Laslett, A. M., \& Dietze, P. (2008). Individual and community correlates of young people's high-risk drinking in Victoria, Australia. Drug Alcohol Depend, 98, 241-248. http://dx.doi. org/10.1016/j.drugalcdep.2008.06.002

Lyvers, M., Hasking, P., Hani, R., Rhodes, M., \& Trew, E. (2009). Drinking motives, drinking restraint and drinking behavior among young adults. Addictive Behaviors, 35, 116-122. http://dx.doi.org/10.1016/j. addbeh.2009.09.011

Mäkelä, K., \& Mustonen, H. (2000). Relationships of drinking behavior, gender and age with reported negative and positive experiences related to drinking. Addiction, 95, 727-736. http://dx.doi. org/10.1046/j.1360-0443.2000.9557278.x

March, J. C., Prieto, M. Á., Danet, A., Escudero, M., López, M., \& Luque, N. (2010). El consumo de alcohol en los adolescentes: una aproximación cualitativa desde los docentes [Alcohol use in adolescents: a qualitative approach from teachers]. Trastornos Adictivos, 12(2), 65-71. http://dx.doi. org/10.1016/S1575-0973(10)70013-1

Mallett, K. A., Marzell, M., Varvi-Weld, L., Turrisi, R., Guttman, K., \& Abar, C. (2011). One-Time or Repeat Offenders? An Examination of the Patterns of Alcohol-Related Consequences Experienced by College Students Across the Freshman Year. Addictive Behaviors, 36, 508-511. http://dx.doi. org/10.1016/j.addbeh.2010.12.022

Martens, M. P., Ferrier, A. G., Sheehy, M. J., Corbett, K., Anderson, D. A., \& Simmons, A. (2005). Development of the protective behavioral strategies survey. Journal of Studies on Alcohol, 66, 698-705.

Martens, M., Rocha, T., Martin, J., \& Serrao, H. (2008). Drinking motives and college students: Further examination of a four-factor model. Journal of Consulting Psychology, 55(2), 289-295. http://dx.doi. org/10.1037/0022-0167.55.2.289

Martin, J. L. (2011). Examination of the interaction of drinking motives and personality on alcohol use and alcohol-related problems among college students. (Order No. 3464655, State University of New York at Albany). ProQuest Dissertations and Theses, 157. Recuperado de http://search.proquest.com/docvie w/886751150?accountid=14777. $(886751150)$.
McCabe, S. E. (2002). Gender differences in collegiate risk factors for heavy episodic drinking. J Stud Alcohol, 63, 49-56.

Ministerio de Sanidad y Consumo (MSC) (2008). Prevención de los problemas derivados del alcohol. $1^{\underline{a}}$ Conferencia de prevención y promoción de la salud en la práctica clínica en España [Prevention of alcohol problems. 1st Conference of prevention and health promotion in clinical practice in Spain]. Madrid: Ministerio de Sanidad y Consumo.

Mooney, D. K., Fromme, K., Kivlahan, D. R., \& Marlatt, G. A. (1987). Correlates of alcohol consumption: sex, age, and expectancies relate differentially to quantity and frequency. Addictive Behaviors, 12, 235-240. http://dx.doi.org/10.1016/03064603(87)90033-5

Motos, P. (2013). Determinantes del consumo intensivo de alcohol en jóvenes universitarios (Tesis doctoral). Valencia: Universitat de València.

National Institute on Alcohol Abuse and Alcoholism (NIAAA) (2004). Council approves definition of binge drinking. NIAAA Newsletter, 3. Retrieved from http://pubs.niaaa.nih.gov/publications/Newsletter/winter2004/Newsletter_Number3.pdf

Neighbors, C., Lee, C. M., Lewis, M. A., Fossos, N., \& Larimer, M. E. (2007). Are Social Norms the Best Predictor of Outcomes Among Heavy-Drinking College Students?. J Stud Alcohol Drugs, 68(4), 556-565.

Neighbors, C., Walker, D. D., \& Larimer, M. E. (2003). Expectancies and evaluations of alcohol effects among college students: Self-determination as a moderator. Journal of Studies on Alcohol, 64, 292-300.

Nolen-Hoeksema S. (2004). Gender differences in risk factors and consequences for alcohol use and problems. Clinical Psychological Review, 24, 981-1010. http://dx.doi.org/10.1016/j.cpr.2004.08.003

Observatorio Español sobre Drogas (OED) (2011). Encuesta Estatal sobre el uso de drogas en estudiantes de enseñanzas secundarias (ESTUDES) [National Survey on drug use in secondary students]. Madrid: Ministerio de Sanidad y Política Social (PNSD).

Observatorio Español sobre Drogas (OED) (2013). Encuesta Estatal sobre el uso de drogas en estudiantes de enseñanzas secundarias (ESTUDES) [National 
Survey on drug use in secondary students]. Madrid: Ministerio de Sanidad y Política Social (PNSD).

O'Malley, P. M., \& Johnston, LL. D. (2002). Epidemiology of Alcohol and Other Drug Use among American College Students. Journal of Studies on Alcohol, 14, 23-39.

Parada, M., Corral, M., Caamaño-Isorna, F., Mota, N., Crego, A., Rodríguez, S., \& Cadaveira, F. (2011). Definición del concepto de consumo intensivo de alcohol adolescente (binge drinking) [Definition of teenage intensive alcohol consumption]. Adicciones, 23, 53-63.

Pilatti, A., Caneto, F., Garimaldi, J. A., Del Valle, B., \& Pautassi, R. M. (2013). Contribution of Time of Drinking Onset and Family History of Alcohol Problems in Alcohol and Drug Use Behaviors in Argentinean College Students. Alcohol and Alcoholism, 49, 128-137. http://dx.doi.org/10.1093/ alcalc/agt176

Pitkänen, T., Lyyra, A. L., \& Pulkkinen, L. (2005). Age of onset of drinking and the use of alcohol in adulthood: a follow-up study from age 8-42 for females and males. Addiction 100, 652-61. http://dx.doi. org/10.1111/j.1360-0443.2005.01053.x

Ray, A. E., Turrisi, R., Abar, B., \& Peters, K. E. (2009). Social-cognitive correlates of protective drinking behaviors and alcohol-related consequences in college students. Addictive Behaviors, 34, 911-917. http://dx.doi.org/10.1016/j.addbeh.2009.05.016

Read, J. P., Wood, M. D., Lejuez, C. W., Palfai, T. P., \& Slack, M. (2004). Gender, alcohol consumption, and differing alcohol expectancy dimensions in college drinkers. Experimental and Clinical Psychopharmacology, 12, 298-308. http://dx.doi. org/10.1037/1064-1297.12.4.298

Rodríguez-Martos, A., Gual, A., \& Llopis, J. J. (1999). La unidad de bebida estándar como registro simplificado del consumo de bebidas alcohólicas y su determinación en España [The "Standard drink unit" as a simplified recording system of alcohol consumption and its measurement in Spain]. Medicina Clínica, 112(12), 446-450.

Rossow, I., \& Kuntsche, E. (2013). Early onset of drinking and risk of heavy drinking in young adulthooda 13-year prospective study. Alcoholism: Clinical and Experimental Research, 37(1), E297-E304. http://dx.doi.org/10.1111/j.1530-0277.2012.01924.x

Shield, K. D., Gmel, G., Patra, J., \& Rehm, J. (2012). Global burden of injuries attributable to alcohol consumption in 2004: a novel way of calculating the burden of injuries attributable to alcohol consumption. Population Health Metrics, 9, 1-14.

Valdivia, I., \& Stewart, S. H. (2005). Further examination of the psychometric properties of the Comprehensive Effects of Alcohol Questionnaire. Cognitive Behaviour Therapy, 34, 22-33. http://dx.doi. org/10.1080/16506070410001009

Warner, L. A., White, H. R., \& Johnson, V. L. (2007). Alcohol initiation experiences and family history of alcoholism as predictors of problem drinking trajectories. J Stud Alcohol, 68, 56-65.

Wechsler, H., Dowdall, G. W., Davenport, A., \& Castillo, S. (1995). Correlates of student binge drinking. American Journal of Public Health, 85, 921-926. http://dx.doi.org/10.2105/AJPH.85.7.921

Wechsler, H., \& Nelson, T. F. (2010). Will increasing alcohol availability by lowering the minimum legal drinking age decrease drinking and related consequences among youths? American Journal of Public Health, 100, 986-992. http://dx.doi.org/10.2105/ AJPH.2009.178004

White, A. M., Kraus, C. L., \& Swartzwelder, H. S. (2006). Many College Freshman Drink at Levels Far Beyond the Binge Threshold. Alcoholism: Clinical and Experimental Research, 30, 1006-1010. http://dx.doi.org/10.1111/j.1530-0277.2006.00122.x 